PESQUIMAT, Revista de la F.C.M. de la

Universidad Nacional Mayor de San Marcos

Vol. XIII N², pp. 21-38, Lima - Perú, Diciembre 2010

\title{
CONTROL NO LINEAL EN LA FRONTERA DE CRISTALES CÚBICOS
}

Yolanda S. Santiago Ayala ${ }^{1}$, Victor R. Cabanillas Zannini ${ }^{2}$ \& Santiago C. Rojas Romero ${ }^{3}$

Resumen: Usando la Teoría de Operadores maximales monótonos y el Teorema de Minty-Browder, probamos la existencia de solución global de un sistema elásticodinámico relativo a cristales cúbicos, al cual suministramos un control no lineal $f$. También, usando técnicas multiplicativas, resultados de Dautry R. - Lions J. L., desigualdades integrales, el Principio de D. L. Russel, el método de W. Liu y adaptando el método de F. Conrad y B. Rao en el cálculo de las estimativas, mostramos que la energía asociada al sistema decae a cero cuando $t \rightarrow+\infty$.

Palabras clave: Estabilidad de un sistema elástico-dinámico, Cristales cúbicos, El Teorema de Minty-Browder.

\section{NONLINEAR CONTROL ON THE BOUNDARY OF CUBIC CRYSTALS}

\begin{abstract}
Using monotone maximal operators Theory and the Minty-Browder theorem, we prove the existence of global solution to the elastic-dynamic system on cubic crystals, which is given a nonlinear control $f$. Also, using multiplicative techniques, results of R. Dautry. - J. L. Lions, integral inequalities, Russel' Principle, the W. Liu method, and adapting the F. Conrad and B. Rao method in the calculation of the estimates, we show that the energy associated to the system decays to zero when $t \rightarrow+\infty$.
\end{abstract}

Key words: Stability of an elastic-dynamic system, Cubic Crystals, Minty-Browder Theorem.

\footnotetext{
${ }^{1}$ UNMSM, Facultad de Ciencias Matemáticas, e-mail: ysantiagoa@unmsm.edu.pe

${ }^{2}$ UNMSM, Facultad de Ciencias Matemáticas, e-mail: vcabanillasz@unmsm.edu.pe

${ }^{3}$ UNMSM, Facultad de Ciencias Matemáticas, e-mail: srojasr@unmsm.edu.pe
} 


\section{Introducción}

Estudiaremos el sistema de elasticidad relativo a cristales cúbicos estabilizado por un control no lineal en la frontera.

Existen dos trabajos de Lagnese, una de las cuales, i.e. [9] obtiene resultados de decrecimiento uniforme de la energía del sistema elástico con control lineal f(u') mas condiciones técnicas sobre el tensor de elasticidad, pero esto no es posible aplicar al sistema elástico homogéneo Isotrópico Lineal (SEHIL). El segundo artículo [10] consigue estimativas de decrecimiento uniforme de la energía para el SEHIL bidimensional con control no lineal f(u') mas un suavizante lineal. Por otro lado, Komornik en [8] mostró el resultado [10] sin el suavizante lineal para el caso $\mathrm{N}=2,3$, sometidos a una fuerza que depende linealmente de la velocidad.

Suponer que $\eta>\lambda+\mu$ esta fisicamente verificado para: Fe, AL, Diamond, LiF, NaCL, KCL, $\mathrm{Na} \mathrm{Br}, \mathrm{KI}$ y Ag CL. El Fe tiene $\gamma=-0.2$. El resto de estos cristales cúbicos tienen $\gamma>0$. Luego nuestro problema tiene sentido, y para complementar podemos citar Feynman [6] y Ciarlet [2]. Es importante citar a Dafermos [4], uno de los pioneros en el estudio del comportamiento asintótico de soluciones de ecuaciones de evolución no lineal. Podemos citar también los siguientes trabajos [15], [16], [17] y [18], donde se abordan el comportamiento asintótico de algunos sistemas de evolución.

\section{Preliminares}

Sea $V$ un espacio de Banach, Reflexivo y $T: V \longrightarrow V^{\prime}$ una aplicación.

Definición 2.1. T es monótono si $\left\langle T v, v>_{V^{\prime}, V} \geq 0 . \forall v \in V\right.$.

Definición 2.2. T es coercivo si $\frac{\langle T v, v\rangle_{V^{\prime}, V}}{\|v\|_{V}} \longrightarrow+\infty$, cuando $\|v\|_{V} \rightarrow+\infty$

Definición 2.3. $T$ es hemicontinuo si $\forall u, v, w \in V$ vale

$$
\begin{aligned}
T: \mathbb{R} & \longrightarrow \mathbb{R} \\
\lambda & \longrightarrow<T(u+\lambda v), w>_{V^{\prime}, V}
\end{aligned}
$$

es continua.

Definición 2.4. $T$ es limitado si existe $C>0$ tal que $\|T v\| \leq C, \forall v \in V$.

Teorema 2.1 (MINTY-BROWDER). Sea $V$ un espacio de Banach, Reflexivo (Separable), $T: V \longrightarrow V^{\prime}$ un operador hemicontinuo, monótono, limitado y coercivo (HEMOLICO) entonces $T$ es sobreyectivo,

Sea el problema de evolución

$$
\begin{gathered}
U^{\prime}+A U=0 \quad \text { en } \mathbb{R}^{+} \\
U(0)=U_{0}
\end{gathered}
$$

donde $A: D(A) \subset H \rightarrow H$ es un operador no necesariamente lineal, en un espacio de Hilbert $H$. 
Definición 2.5. Diremos que $A$ es maximal monótono si son satisfechas las siguientes dos propiedades:

1. A es monótono: $(A U-A V, U-V)_{H} \geq 0, \forall U, V \in D(A)$.

2. $I+A$ es sobreyectiva: $\operatorname{Im}(I+A)=H$.

Teorema 2.2 (Hille-Yosida). Sea A un operador monónotono maximal en un espacio de Hilbert $H$. Entonces, para cada $U_{0} \in \overline{D(A)}$ el problema (1) tiene una única solución

$$
U \in C\left(\mathbb{R}^{+}, H\right)
$$

(definido de un modo adecuado).

Si $V^{0} \in \overline{D(A)}$ y si $V$ es la correspondiente solución de (1), entonces la función

$$
: t \longrightarrow\|U(t)-V(t)\|_{H}
$$

es no-creciente en $\mathbb{R}^{+}$.

Si $U^{0} \in D(A)$, entonces la solución es mucho mas regular

$$
U \in W^{1, \infty}\left(\mathbb{R}^{+}, H\right)
$$

y la función:

$$
: t \longrightarrow\|A(U)\|_{H}
$$

está definida en todo punto y es no-creciente en $\mathbb{R}^{+}$.

Lema 2.1 (Desigualdad Integral). Sea $E: \mathbb{R}^{+} \longrightarrow \mathbb{R}^{+}$una función no-creciente y supongamos que exista una constante $T>0$ tal que

$$
\int_{t}^{+\infty} E(s) d s \leq T E(t), \quad \forall t \geq 0
$$

entonces

$$
E(t) \leq E(0) e^{1-\frac{t}{T}}, \quad \forall t \geq T .
$$

Se verifica fácilmente que la desigualdad (7) también es válida para $0 \leq t<T$.

\section{Resultados Principales}

\subsection{Resultado Principal de Existencia de Solución}

Sea $\Omega$ un dominio acotado de $\mathbb{R}^{N}$ de clase $C^{1}, \Gamma=\partial \Omega, \nu=\left(\nu_{1}, \ldots, \nu_{n}\right)$ vector normal unitario sobre $\Gamma$.

Sea $\Gamma_{0}, \Gamma_{1}$ una partición de la frontera $\Gamma$ tal que $\overline{\Gamma_{0}} \cap \overline{\Gamma_{1}}=\emptyset$. Sean las constantes positivas: $\lambda, \mu$ , $\eta$, los parámetros del tensor de elasticidad y definimos $\gamma:=\eta-\lambda-2 \mu$.

Sean $a, l: \Gamma_{1} \rightarrow \mathbb{R}^{+}$funciones de clase $C^{1}$ y vamos ha suponer que $a \not \equiv 0$ si $\Gamma_{0}=\emptyset$.

Sea $g: \mathbb{R} \rightarrow \mathbb{R}$ una función continua no decreciente que se anule en el cero ( i.e. $g(0)=0$ ) tal que $\forall x \in \mathbb{R}|g(x)| \leq C(1+|x|)$, donde $C>0$. 
Observación 3.1. Se observa que $x g(x) \geq 0, \forall x \in \mathbb{R}$.

Consideremos el sistema de evolución $(\mathcal{P})$

$$
\begin{aligned}
& u_{i}^{\prime \prime}-\mu \Delta u_{i}-(\lambda+\mu) \frac{\partial}{\partial x_{i}}(\operatorname{div} u)-\gamma \frac{\partial^{2} u_{i}}{\partial x_{i}}=0 \text { en } \Omega \times \mathbb{R}^{+} \\
& u_{i}=0 \text { en } \Gamma_{0} \times \mathbb{R}^{+} \\
& \mu \partial_{\nu} u_{i}+(\lambda+\mu)(\text { divu }) \nu_{i}+\gamma \frac{\partial u_{i}}{\partial x_{i}} \nu_{i}+a u_{i}+\lg \left(u_{i}^{\prime}\right)=0 \text { en } \Gamma_{1} \times \mathbb{R}^{+} \\
& u_{i}(0)=u_{i}^{o} \quad u_{i}^{\prime}(0)=u_{i}^{1} \text { en } \Omega
\end{aligned}
$$

para $i=1, \ldots, N$.

Usando resultados estandares de la Teoría de Operadores Maximales Monótonos obtenemos el siguiente resultado de existencia y regularidad de solución

Teorema 3.1. Asumamos: $\eta>\lambda+\mu$.

1. Dado $\left(u^{o}, u^{1}\right) \in H_{\Gamma_{0}}^{1}(\Omega)^{N} \times L^{2}(\Omega)^{N}$. El Problema $(\mathcal{P})$ admite una única solución débil

$$
u \in C\left(\mathbb{R}^{+}, H_{\Gamma_{0}}^{1}(\Omega)^{N}\right) \cap C^{1}\left(\mathbb{R}^{+}, L^{2}(\Omega)^{N}\right)
$$

Además, la aplicación $:\left(u^{o}, u^{1}\right) \longrightarrow u$ es continua con respecto a dichas topologías; y la energía de u definida por

$$
E(t):=\frac{1}{2} \int_{\Omega} u^{\prime 2}+\mu|\nabla u|^{2}+(\lambda+\mu)[\operatorname{div}(u)]^{2}+\gamma \sum_{i=1}^{N}\left(\frac{\partial u_{i}}{\partial x_{i}}\right)^{2}+\frac{1}{2} \int_{\Gamma_{1}} a u^{2}
$$

es una función no creciente.

2. Además, si g es globalmente Lipschitziana y si $u^{\circ}, u^{1}$ verifican la siguiente condición fuerte:

$$
\left\{\begin{array}{l}
\forall i=1, \ldots, N \\
u^{o} \in H^{2}(\Omega)^{N} \cap H_{\Gamma_{o}}^{1}(\Omega)^{N}, \quad u^{1} \in H_{\Gamma_{o}}^{1}(\Omega)^{N} \\
\mu \partial_{\nu} u_{i}^{o}+(\lambda+\mu)\left(\text { divu }^{o}\right) \nu_{i}+\gamma \frac{\partial u_{i}^{o}}{\partial x_{i}} \nu_{i}+a u_{i}^{o}+l g\left(u_{i}^{1}\right)=0
\end{array}\right.
$$

Entonces la solución u del problema $\mathcal{P}$ posee la propiedad de regularidad mas fuerte

$$
\begin{aligned}
& u \in L^{\infty}\left(\mathbb{R}^{+}, H^{2}(\Omega)^{N}\right) \\
& u^{\prime} \in L^{\infty}\left(\mathbb{R}^{+}, H_{\Gamma_{o}}^{1}(\Omega)^{N}\right) \\
& u^{\prime \prime} \in L^{\infty}\left(\mathbb{R}^{+}, L^{2}(\Omega)^{N}\right)
\end{aligned}
$$

En este caso u es llamada Solución Fuerte del problema $\mathcal{P}$

\subsection{Resultado Principal de Estabilización}

Sabemos que el Problema $\mathcal{P}$ es disipativo. Supongamos satisfechas las siguientes condiciones:

a) $N \geq 3$

b) Sea Sea $x_{0} \in \mathbb{R}^{N}$, definimos $m_{x_{0}}=m(x):=x-x_{0}$, tal que

$$
\begin{aligned}
& m(x) \cdot \nu(x) \leq 0 \text { sobre } \Gamma_{0} \\
& m(x) \cdot \nu(x)>0 \text { sobre } \Gamma_{1}
\end{aligned}
$$


c) $R:=\sup _{x \in \bar{\Omega}}|m(x)|$

d) $\lambda, \mu, \eta$ satisfacen la desigualdad:

$$
\eta>\lambda+\mu
$$

luego $\mu+\gamma>0$, desde que $\gamma:=\eta-\lambda-2 \mu$ y $\mu+\gamma=\eta-\lambda-\mu$.

Observación 3.2. El caso homogéneo Isotrópico corresponde $a(\gamma=0, \eta=\lambda+2 \mu)$.

e)

$$
\mathcal{E}:=\inf \{\mu, \mu+\gamma\}=\inf \{\mu, \eta-\lambda-\mu\}
$$

Por lo tanto $0<\mathcal{E}$

f) $\alpha, \beta: \Gamma_{1} \longrightarrow \mathbb{R}_{+}$, son funciones continuas y estrictamente positivas.

Definimos las siguientes funciones: $a, l: \Gamma_{1} \longrightarrow \mathbb{R}$, mediante:

$$
\left.\begin{array}{l}
a(x):=\alpha(x) m(x) \cdot \nu(x) \\
l(x):=\alpha(x) m(x) \cdot \nu(x)
\end{array}\right\} .
$$

Teorema 3.2. Supongamos que se verifican: (18) , (19) , (20)

1. Supongamos que existan las constantes: $p>1, c_{1}>0, c_{2}>0, c_{3}>0, c_{4}>0$ tal que

$$
\begin{array}{rr}
c_{1}|x|^{p} \leq|g(x)| \leq c_{2}|x|^{\frac{1}{p}} & \text { si }|x| \leq 1 \\
c_{3}|x| \leq|g(x)| \leq c_{4}|x| & \text { si }|x|>1
\end{array}
$$

Entonces, dado $\left(u^{0}, u^{1}\right) \in H_{\Gamma_{0}}^{1}(\Omega)^{N} \times L^{2}(\Omega)^{N}$

(i.e. Debido al Teorema 3.1 existe la solución débil u tal que $u \in C\left(\mathbb{R}_{+}, V\right) \cap C^{1}\left(\mathbb{R}_{+}, H\right)$ y $E=\frac{1}{2}\left\|\left(u, u^{\prime}\right)\right\|_{V \times H}^{2}$ es decreciente.)

La solución de $(\mathcal{P})$ satisface la siguiente estimativa

$$
\text { Decaimiento Polinomial de la Energía: } E(t) \leq C t^{\frac{-2}{p-1}}, \forall t>0
$$

donde $C$ es una constante que depende de la energía inicial $E(0)$ y del modo continuo.

2. Si existen las constantes positivas c y $c^{\prime}$ tal que

$$
c|x| \leq|g(x)| \leq c^{\prime}|x|, \forall x \in \mathbb{R}
$$

Observación 3.3. Si $g(x)=x$ entonces $c=c^{\prime}=1$.

Entonces, existe $w>0$ tal que, la solución débil de $(\mathcal{P})$ satisface la siguiente estimativa

$$
\text { Decaimiento exponencial de la Energía: } E(t) \leq E(0) e^{1-w t}, \forall t>0
$$

Además, si definimos a y $l$ de la siguiente forma:

$$
a=\tilde{a}:=\frac{N-1}{2 R^{2}} \xi m \cdot \nu \text { y } l=\tilde{l}:=\frac{\sqrt{\xi}}{R c^{\prime}} m \cdot \nu
$$

Entonces, la energía de la solución débil de $(P)$ satisface:

$$
\text { Decaimiento Exponencial: } \quad E(t) \leq E(0) e^{1-\frac{c \sqrt{\xi}}{c+c^{\prime} R} t}, \forall t \geq 0
$$




\section{Planteamiento y Solución del Problema}

\subsection{Existencia de Solución}

Definamos $H:=L^{2}(\Omega)^{N}$, que es un espacio de Hilbert Separable, con norma:

$$
\|v\|_{L^{2}(\Omega)^{N}}^{2}=\int_{\Omega} v^{2}=\sum_{i=1}^{N} \int_{\Omega} v_{i}^{2}=\int_{\Omega} v_{i} v_{i} \text { notación de indices repetidos }
$$

También defina

$$
V:=H_{\Gamma_{0}}^{1}(\Omega)^{N}=\left\{v \in H^{1}(\Omega)^{N}, v=0 \text { sobre } \Gamma_{0}\right\}
$$

que es un espacio de Hilbert con la norma (que viene de un producto interno):

$$
\|v\|_{V}:=\int_{\Omega} \mu|\nabla v|^{2}+(\lambda+\mu)(\operatorname{div} v)^{2}+\gamma \sum_{i=1}^{N}\left(\frac{\partial v_{i}}{\partial x_{i}}\right)^{2} d x+\int_{\Gamma_{1}} a v^{2}
$$

y esta norma es equivalente a la norma $\|\cdot\|_{\left(H^{1}(\Omega)\right)^{N}}$ desde que $\mu+\gamma>0$.

y $V \hookrightarrow H$ inmerso compactamente.

Sea $A$ el operador definido por:

$$
\begin{aligned}
A: V & \longrightarrow V^{\prime} \\
v & \longrightarrow A v, \text { donde }<A v, w>_{V^{\prime} V}:=<v, w>_{V} \forall w \in V
\end{aligned}
$$

entonces $A$ es lineal y acotado. En efecto $|\langle A v, w\rangle|=|\langle v, w\rangle| \leq\|v\|\|w\|$ entonces $|A v| \leq\|v\|$.

Sea $B$ el operador definido por:

$$
\begin{aligned}
B: V & \longrightarrow V^{\prime} \\
v & \longrightarrow B v, \text { donde }\left\langle B v, w>_{V^{\prime} V}:=\int_{\Gamma_{1}} l g(v) \cdot w=\sum \int_{\Gamma_{1}} l g\left(v_{i}\right) \cdot w_{i}, \forall w \in V\right.
\end{aligned}
$$

entonces $B$ en general no es lineal. Se observa que $B$ esta bien definido debido a la inyección de $H^{1}(\Omega)$ en $L^{2}(\Gamma)$ y

$$
V \subset\left[H^{1}(\Omega)\right]^{N} \hookrightarrow\left[H^{\frac{1}{2}}(\Gamma)\right]^{N} \hookrightarrow\left[L^{2}(\Gamma)\right]^{N}
$$

Observación 4.1. Sean $w=\left(w_{1}, \ldots, w_{N}\right), v=\left(v_{1}, \ldots, v_{N}\right) \in V$ entonces

i) $g\left(v_{i}\right) \in L^{2}(\Gamma)$

ii) $w_{i} \in L^{2}(\Gamma)$

En efecto,

i) Como $v_{i}=w_{i}=0$ en $\Gamma_{0}$, debido al trazo tenemos que $v_{i}, w_{i} \in H^{1}(\Omega) \hookrightarrow H^{\frac{1}{2}}(\Gamma) \hookrightarrow L^{2}(\Gamma)$.

ii) Sabemos que $\left|g\left(v_{i}\right)\right| \leq C\left(1+\left|v_{i}\right|\right)$, luego $\left|g\left(v_{i}\right)\right|^{2} \leq \tilde{C}\left(1+\left|v_{i}\right|^{2}\right)$.

$$
\begin{aligned}
\int_{\Gamma_{1}}\left|g\left(v_{i}\right)\right|^{2}=\int_{\Gamma}\left|g\left(v_{i}\right)\right|^{2} & \leq \tilde{C} \int_{\Gamma}\left(1+\left|v_{i}\right|^{2}\right) \\
& \leq \tilde{C} \mu(\Gamma)+\int_{\Gamma}\left|v_{i}\right|^{2} \\
& =\hat{C}+\int_{\Gamma}\left|v_{i}\right|^{2}<+\infty
\end{aligned}
$$

i.e. $g\left(v_{i}\right) \in L^{2}\left(\Gamma_{1}\right)$. 
Por otro lado,

$$
\begin{aligned}
\left|\int_{\Gamma_{1}} l g\left(v_{i}\right) \cdot w_{i}\right| & \leq \int_{\Gamma_{1}}|l|\left|g\left(v_{i}\right)\right|\left|w_{i}\right| \\
& \leq C\left|g\left(v_{i}\right)\right|_{L^{2}\left(\Gamma_{1}\right)}\left|w_{i}\right|_{L^{2}\left(\Gamma_{1}\right)} \\
& \leq C\left|g\left(v_{i}\right)\right|_{L^{2}(\Gamma)}\left|w_{i}\right|_{L^{2}(\Gamma)}<+\infty
\end{aligned}
$$

luego,

$$
\left|<B v, w>_{V^{\prime} V}\right| \leq \sum_{i=1}^{N}\left|\int_{\Gamma_{1}} \lg \left(v_{i}\right) \cdot w_{i}\right| \leq C \sum_{i=1}^{N}\left|g\left(v_{i}\right)\right|_{L^{2}(\Gamma)}\left|w_{i}\right|_{L^{2}(\Gamma)}<\infty
$$

Luego $B$ está bien definida.

Por otro lado,

$$
\begin{aligned}
\left|<B v, w>_{V^{\prime} V}\right| & \leq C \sum_{i=1}^{N}\left|g\left(v_{i}\right)\right|_{L^{2}(\Gamma)}\left|w_{i}\right|_{L^{2}(\Gamma)} \\
& \leq C \sum_{i=1}^{N}\left\{\hat{C}+\int_{\Gamma_{1}}\left|v_{i}\right|^{2}\right\}\left|w_{i}\right|_{L^{2}(\Gamma)} \\
& \leq \check{c}\left\{1+\|v\|_{V}^{2}\right\}\|w\|_{V}
\end{aligned}
$$

Así, $\|B v\| \leq \check{c}\left\{1+\|v\|_{V}^{2}\right\}$, i.e. $B v \in V^{\prime}$ y $B$ es acotada.

Supongamos que $u$ sea solución del problema $(\mathcal{P})$. Sea $v \in V$. Multiplicando (8) por $v_{i}$, integrando por partes sobre $\Omega$ obtenemos una formulación débil del problema $(\mathcal{P})$,

$$
\begin{aligned}
0= & \int_{\Omega}\left[u_{i}^{\prime \prime}-\mu \Delta u_{i}-(\lambda+\mu) \frac{\partial}{\partial x_{i}}(\operatorname{div} u)-\gamma \frac{\partial^{2} U_{i}}{\partial x_{i}}\right] v_{i} d x \\
= & \int_{\Omega} u_{i}^{\prime \prime} v_{i}-\mu \Delta u_{i} v_{i}-(\lambda+\mu) \frac{\partial}{\partial x_{i}}(\operatorname{div} u) v_{i}-\gamma \frac{\partial^{2} U_{i}}{\partial x_{i}} v_{i} d x \\
= & \int_{\Omega} u_{i}^{\prime \prime} v_{i}+\mu \int_{\Omega} \nabla u_{i} \nabla v_{i}+(\lambda+\mu) \int_{\Omega} \operatorname{div} u \frac{\partial v_{i}}{\partial x_{i}}+\gamma \int_{\Omega} \frac{\partial u_{i}}{\partial x_{i}} \frac{\partial v_{i}}{\partial x_{i}} \\
& -\mu \int_{\Gamma_{1}} v_{i} \frac{\partial u_{i}}{\partial \nu}-(\lambda+\mu) \int_{\Gamma_{1}} \operatorname{div} u v_{i} \nu_{i}-\gamma \int_{\Gamma_{1}} \frac{\partial u_{i}}{\partial x_{i}} v_{i} \nu_{i} \\
= & \int_{\Omega}\left[u_{i}^{\prime \prime} v_{i}+\mu \nabla u_{i} \nabla v_{i}+(\lambda+\mu) \operatorname{div} u \frac{\partial v_{i}}{\partial x_{i}}+\gamma \frac{\partial u_{i}}{\partial x_{i}} \frac{\partial v_{i}}{\partial x_{i}}\right] \\
& -\int_{\Gamma_{1}}\left[\mu \frac{\partial u_{i}}{\partial \nu}+(\lambda+\mu) \operatorname{div} u \nu_{i}+\gamma \frac{\partial u_{i}}{\partial x_{i}} \nu_{i}\right] v_{i}
\end{aligned}
$$

Sumando sobre $i$, obtenemos $0=\left\langle u^{\prime \prime}, v>_{V^{\prime} V}+<A u, v>_{V^{\prime} V}+<B u^{\prime} v>_{V^{\prime} V}\right.$, i.e.

$$
0=<u^{\prime \prime}+A u+B u^{\prime}, v>_{V^{\prime} V}, \forall v \in V
$$

Tomamos, $U=\left(u_{1}, u_{2}\right):=\left(u, u^{\prime}\right)$.

Definimos el operador $\mathcal{A}$

$$
\mathcal{A} U=\left(-U_{2}, A U_{1}+B U_{2}\right)
$$

entonces el Problema $(\mathcal{P})$ se escribe como

$$
\begin{aligned}
U^{\prime}+\mathcal{A U} & =0 \quad \text { sobre } \mathbb{R}^{+} \\
U(0) & =\left(u_{0}, u_{1}\right)
\end{aligned}
$$


Observación 4.2. Si u es solución suficientemente regular de (8)-(11), entonces $U$ y $\mathcal{A} U$ estan en $V \times H$.

\section{Definición 4.1.}

$$
D(\mathcal{A}):=\left\{U \in V \times V, A U_{1}+B U_{2} \in H\right\}
$$

Definición 4.2. La solución débil del Problema (P) viene a ser la proyección sobre la primera componente de la solución de (33)

Proposición 4.1. $\mathcal{A}$ es un operador maximal monótono de $V \times H$.

Prueba. $\mathcal{A}$ es monótono:

Sea $U, V \in D(\mathcal{A})$ con $U=\left(U_{1}, U_{2}\right)$ y $V=\left(V_{1}, V_{2}\right)$, probaremos que $<\mathcal{A} U-\mathcal{A} V, U-V>_{V \times H} \geq 0$.

$$
\begin{aligned}
< & \mathcal{A} U-\mathcal{A} V, U-V>_{V \times H} \\
:= & <\left(-U_{2}, A U_{1}+B U_{2}\right)-\left(-V_{2}, A V_{1}+B V_{2}\right),\left(U_{1}-V_{1}, U_{2}-V_{2}\right)>_{V \times H} \\
= & <\left(-U_{2}+V_{2}, A V_{1}+B U_{2}-A V_{1}-B V_{2}\right),\left(U_{1}-V_{1}, U_{2}-V_{2}\right)>_{V \times H} \\
= & <-U_{2}+V_{2}, U_{1}-V_{1}>_{V}+<A U_{1}+B U_{2}-A V_{1}-B V_{2}, U_{2}-V_{2}>_{H} \\
& \text { Como }<A U_{1}-A V_{1}, U_{2}-V_{2}>_{V^{\prime} V}=<U_{1}-V_{1}, U_{2}-V_{2}>_{V}, \\
= & <-U_{2}+V_{2}, U_{1}-V_{1}>_{V}+<A U_{1}-A V_{1}, U_{2}-V_{2}>_{V^{\prime} V}+ \\
< & B U_{2}-B V_{2}, U_{2}-V_{2}>_{V^{\prime} V} \\
= & \int_{\Gamma_{1}} l\left[g\left(U_{2}\right)-g\left(V_{2}\right)\right] \cdot\left(U_{2}-V_{2}\right) \\
\geq & 0
\end{aligned}
$$

puesto que si $g$ es creciente entonces $[g(x)-g(y)](x-y) \geq 0$, en efecto, $\int_{\Gamma_{1}} l\left[g\left(U_{2}\right)-g\left(V_{2}\right)\right]$. $\left(U_{2}-V_{2}\right)=\sum_{i=1}^{N} \int_{\Gamma_{1}} l\left[g\left(U_{2}^{i}\right)-g\left(V_{2}^{i}\right)\right] \cdot\left(U_{2}^{i}-V_{2}^{i}\right)$ y si $U_{2}^{i}>V_{2}^{i}$ implica $g\left(U_{2}^{i}\right) \geq\left(V_{2}^{i}\right)$ entonces $g\left(U_{2}^{i}\right)-\left(V_{2}^{i}\right) \geq 0$, luego $\left[g\left(U_{2}^{i}\right)-g\left(V_{2}^{i}\right)\right] \cdot\left(U_{2}^{i}-V_{2}^{i}\right) \geq 0$ y como $l>0$, se sigue.

Acontinuación estudiaremaos el caracter maximal del Operador $\mathcal{A}$.

$I+\mathcal{A}: D(\mathcal{A}) \longrightarrow V \times H$ es sobreyectivo:

Sea $\left(w_{1}, w_{2}\right)=W \in V \times H$ probaremos que existe $U=\left(U_{1}, U_{2}\right) \in D(\mathcal{A})$ tal que $(I+\mathcal{A}) U=W$. Esto es que exista $U=\left(U_{1}, U_{2}\right) \in D(\mathcal{A})$ tal que

$$
\begin{aligned}
U_{1}-U_{2} & =w_{1} \\
U_{2}+A U_{1}+B U_{2} & =w_{2}
\end{aligned}
$$

Si $U_{1}:=w_{1}+U_{2}$, reemplazando esto en (35) tenemos $w_{2}=U_{2}+A\left(w_{1}+U_{2}\right)+B U_{2}=$ $U_{2}+A w_{1}+A U_{2}+B U_{2}$, de donde $V^{\prime} \ni w_{2}-A w_{1}=U_{2}+A U_{2}+B U_{2}=(I+A+B) U_{2}$

Todo se reduce a probar la sobreyectividad de $I+A+B: V \longrightarrow V^{\prime}$.

Lema 4.1. El Operador $I+A+B: V \longrightarrow V^{\prime}$ es sobreyectivo.

Prueba. Usaremos el Teorema de Minty-Browder. Es decir probaremos que el Operador es Monótono, Coercivo, Hemicontinuo y acotado. 
i) $I+A+B$ es Monótono.- Sea $v \in V$,

$$
\begin{aligned}
<(I+A+B) v, v>_{V^{\prime} V} & =<v, v>_{V^{\prime} V}+<A v, v>_{V^{\prime} V}+<B v, v>_{V^{\prime} V} \\
& =\|v\|_{H^{2}}^{2}+<v, v>_{V}+<B v, v>_{V^{\prime} V} \\
& =\|v\|_{H}^{2}+<v, v>_{V}+\int_{\Gamma} l g(v) v \\
& =\|v\|_{H}^{2}+\|v\|_{V}^{2}+\sum_{i=1}^{N} \int_{\Gamma} \underbrace{l}_{>0} \underbrace{g\left(v_{i}\right) v_{i}}_{\geq 0} \\
& \geq 0
\end{aligned}
$$

ii) $I+A+B$ es Coercivo.- Usando la identidad del item anterior, tenemos que $<(I+A+$ $B) v, v>_{V^{\prime} V} \geq\|v\|_{V}^{2}, \forall v \in V$, luego

$$
\frac{<(I+A+B) v, v>_{V^{\prime} V}}{\|v\|_{V}} \geq\|v\|_{V}
$$

Tomando límite cuando $\|v\|_{V} \rightarrow+\infty$, tenemos $\frac{\left\langle(I+A+B) v, v{\dot{V^{\prime} V}}\right.}{\|v\|_{V}} \longrightarrow+\infty$.

iii) $I+A+B$ es Hemicontinua.- Probaremos que

$$
\lambda \longrightarrow<(I+A+B)(u+\lambda v), w>_{V^{\prime} V}=<(I+A)(u+\lambda v), w>_{V^{\prime} V}+<B(u+\lambda v), w>_{V^{\prime} V}
$$

es continua. Obviamente $\lambda \longrightarrow<(I+A)(u+\lambda v), w>_{V^{\prime} V}$ es continua. Resta mostrar que $\lambda \longrightarrow<B(u+\lambda v), w>_{V^{\prime} V}$ es continua.

En efecto, sea $\lambda_{n} \rightarrow \lambda$ luego, $u_{i}+\lambda_{n} v_{i} \rightarrow u_{i}+\lambda v_{i}$ y como $g$ es continua, entonces $\underbrace{g\left(u_{i}+\lambda_{n} v_{i}\right)}_{g_{n}:=} \longrightarrow g\left(u_{i}+\lambda v_{i}\right)$, luego $g_{n} w_{i} l \longrightarrow g\left(u_{i}+\lambda v_{i}\right) w_{i} l$. Como $\left|g_{n} w_{i} l\right| \leq C\left(1+\mid u_{i}+\right.$ $\left.\lambda_{n} v_{i} \mid\right)\left|w_{i} l\right| \leq C\left(1+\epsilon+\left|u_{i}+\lambda v_{i}\right|\right)\left|w_{i} l\right| \in L^{1}$, usando el Teorema de la Convergencia Dominada de Lebesgue obtenemos

$$
\begin{aligned}
<B\left(u+\lambda_{n} v\right), w>_{V^{\prime} V} & =\int_{\Gamma} l g\left(u+\lambda_{n} v\right) w \\
& =\sum_{i=1}^{N} \int_{\Gamma} l g\left(u_{i}+\lambda_{n} v_{i}\right) w_{i} \\
& \downarrow \quad \text { cuando } N \rightarrow+\infty \\
<B(u+\lambda v), w>_{V^{\prime} V} & =\sum_{i=1}^{N} \int_{\Gamma} l g\left(u_{i}+\lambda_{i}\right) w_{i}
\end{aligned}
$$

iv) $I+A+B$ es acotado.- En efecto pues se mostró que $I+A$ y $B$ son acotados.

Usando el Teorema de Minty- Browder, concluimos que $I+A+B$ es sobreyectiva.

El Teorema de Hille - Yosida nos permite aseverar la existencia de solución débil de 33, y por lo tanto la existencia de solución débil de $(\mathcal{P})$ satisfaciendo 12 .

Se deduce fácilmente que

Observación 4.3. La energía de la solución $E(u)=\frac{1}{2}\|U\|_{V \times H}^{2}$ es decreciente. 
Definición 4.3. Sean $u_{0}, u_{1} \in V$ satisfaciendo (14), definamos

$$
\begin{aligned}
\mathcal{D}_{0}=: & \left\{\left(u_{0}, u_{1}\right) \in\left[H^{2}(\Omega) \cap V\right] \times V\right. \text { tal que } \\
& \mu \partial_{\nu} u_{i}^{2}+(\lambda+\mu) d i v u^{0} \nu_{i}+\gamma \frac{\partial u_{i}^{0}}{\partial x_{i}} \nu_{i}+a u_{i}^{0}+\lg \left(u_{i}^{1}\right)=0 \text { en } \Gamma_{i} \\
& \text { para } i=1, \ldots N\}
\end{aligned}
$$

Entonces $\left(u^{0}, u^{1}\right) \in \mathcal{D}_{0}$ y además $\mathcal{D}_{0} \subset D(A)$.

$\underline{\mathcal{D}_{0} \subset D(A):}$

$\overline{\text { Sean } u_{0}, u_{1}} \in \mathcal{D}_{0}$ entonces $u^{0} \in V \cap\left(H^{2}\right)^{N}$ y $u^{1} \in V$, luego $u^{0}, u^{1} \in V$. Probaremos que $A u^{0}+B u^{1} \in H \approx H^{\prime}$. Todo se reduce a probar que

$$
\exists C \in \mathbb{R} \text { tal que }\left|<A u^{0}+B u^{1}, w>_{V^{\prime} V}\right| \leq C\|w\|_{H}, \quad \forall u \in V
$$

desde que $V$ es denso en $H$. En efecto, sea $w \in V$,

$$
\begin{aligned}
& <A u^{0}+B u^{1}, w>_{V^{\prime} V}=<A u^{0}, w>_{V^{\prime} V}+<B u^{1}, w>_{V^{\prime} V} \\
& =<u^{0}, w>+\int_{\Gamma_{1}} \lg \left(u^{1}\right) w \\
& =\int_{\Omega} \mu \nabla u^{0} \cdot \nabla w+(\lambda+\mu) d i v u^{0} d i v w+\gamma \sum_{i=1}^{N}\left(\frac{\partial u_{i}^{0}}{\partial x_{i}}\right)\left(\frac{\partial w_{i}}{\partial x_{i}}\right) \\
& +\int_{\Gamma_{1}} a u^{0} w+\int_{\Gamma_{1}} \lg \left(u^{1}\right) w \\
& =\int_{\Omega} \mu \nabla u_{i}^{0} \nabla w_{i}+(\lambda+\mu) d i v u^{0} \cdot \operatorname{div} w+\gamma u_{i, i}^{0} w_{i, i} \\
& +\int_{\Gamma_{1}} a u_{i}^{0} w_{i}+\int_{\Gamma_{1}} l g\left(u_{i}^{1}\right) w_{i} \\
& =-\mu \int_{\Omega} \Delta u_{i}^{0} w_{i}-(\lambda+\mu) \frac{\partial}{\partial x_{i}}\left(\operatorname{div} u^{0}\right) w_{i}-\gamma \frac{\partial^{2} u_{i}^{0}}{\partial x_{i}^{2}} w_{i} \\
& +\mu \int_{\Gamma_{1}} w_{i} \partial_{\nu} u_{i}+(\lambda+\mu) \int_{\Gamma_{1}} \operatorname{divu}^{0} w_{i} \nu_{i}+\gamma \int_{\Gamma_{1}} \frac{\partial u_{i}^{0}}{\partial x_{i}} w_{i} \nu_{i} \\
& +\int_{\Gamma_{1}} a u_{i}^{0} w_{i}+\int_{\Gamma_{1}} l g\left(u_{i}^{1}\right) w_{i} \\
& =\int_{\Omega}-\underbrace{\left(\mu \Delta u_{i}^{0}+(\lambda+\mu) \frac{\partial}{\partial x_{i}}\left(\operatorname{div} u^{0}\right)+\gamma \frac{\partial^{2} u_{i}^{0}}{\partial x_{i}^{2}}\right)}_{\in L^{2}(\Omega)} w_{i} \\
& +\int_{\Gamma_{1}} \underbrace{\left(\mu \partial_{\nu} u_{i}^{0}+(\lambda+\mu) d i v u^{0} \nu_{i}+\gamma \frac{\partial u_{i}^{0}}{\partial x_{i}} \nu_{i}+a u_{i}^{0}+\lg \left(u_{i}^{1}\right)\right)}_{=0} w_{i} \\
& =-\int_{\Omega}\left(\mu \Delta u_{i}^{0}+(\lambda+\mu) \frac{\partial}{\partial x_{i}}\left(\operatorname{div} u^{0}\right)+\gamma \frac{\partial^{2} u_{i}^{0}}{\partial x_{i}^{2}}\right) w_{i}
\end{aligned}
$$

Luego

$$
\begin{gathered}
\left|<A u^{0}+B u^{1}, w>_{V^{\prime} V}\right| \leq\left|\mu \Delta u_{i}^{0}+(\lambda+\mu) \frac{\partial}{\partial x_{i}}\left(d i v u^{0}\right)+\gamma \frac{\partial^{2} u_{i}^{0}}{\partial x_{i}^{2}} \| w_{i}\right| \\
\left.\leq C\left\|u^{0}\right\|_{H^{2}(\Omega}\right)^{N}\|w\|_{H}
\end{gathered}
$$

Se satisface 
i) $\mathcal{D}_{0}$ es denso en $V \times H$, desde que $\mathcal{D}$ es denso en $V$ y en $H$.

ii) $\mathcal{D}(\mathcal{A})$ es denso en $V \times H$, desde que $\mathcal{D}_{0}$ es denso en $H$.

iii) Si $g$ es globalmente Lipschitziana, entonces $\mathcal{D}_{0}=\mathcal{D}(\mathcal{A})$. Usamos Teoría de regularidad elíptica e inmersiones.

Observación 4.4. Si $g$ es globalmente Lipschitziana y continua con $g(0)=0$ entonces $|g(x)| \leq$ $1+C|x|, \forall x \in \mathbb{R}$.

Obtenemos (15)-(16) como consecuencia del Teorema de Hille-Yosida.

\subsection{Estabilización de la Energía}

Nos basamos en la identidad del Lema 4.3. Previamente enunciamos el siguiente resultado.

Lema 4.2.

$$
E(S)-E(T)=\int_{S}^{T} \int_{\Gamma_{1}} l u_{i}^{\prime} g\left(u_{i}^{\prime}\right) d s d t, \forall 0 \leq \dot{S} \leq T<+\infty
$$

Observación 4.5. La igualdad (36) implica que E es absolutamente continua entonces es derivable en casi todo punto y $E^{\prime}(t)=-\int_{\Gamma_{1}} l u_{i}^{\prime} g\left(u_{i}^{\prime}\right) d s$.

Prueba. Multiplicando la ecuación (8) por $u_{i}^{\prime}$ e integrando por partes sobre $\Omega \times[S, T]$ con $0 \leq S \leq T<+\infty$ obtenemos

$$
\begin{aligned}
0= & \int_{S}^{T} \int_{\Omega} u_{i}^{\prime}\left(u_{i}^{\prime \prime}-\mu \Delta u_{i}-(\lambda+\mu) \frac{\partial}{\partial x_{i}}(\operatorname{divu})-\gamma \frac{\partial^{2} u_{i}}{\partial x_{i}{ }^{2}}\right) \\
= & \int_{S}^{T} \int_{\Omega} u_{i}^{\prime} u_{i}^{\prime \prime}+\mu \nabla u_{i}^{\prime} \nabla u_{i}+(\lambda+\mu) \operatorname{div} u \frac{\partial u_{i}^{\prime}}{\partial x_{i}}+\gamma \frac{\partial u_{i}}{\partial x_{i}} \frac{\partial u_{i}^{\prime}}{\partial x_{i}} \\
& \underbrace{-\int_{S}^{T} \int_{\Gamma} u_{i}^{\prime}\left(\mu \partial_{\nu} u_{i}+\left(\operatorname{div} u \nu_{i}\right)(\lambda+\mu)+\gamma \frac{\partial u_{i}}{\partial x_{i}} \nu_{i}\right)}_{J_{1}:=}
\end{aligned}
$$

como $u_{i}^{\prime}=0$ en $\Gamma_{0} \times \mathbb{R}_{+}$entonces

$$
\begin{aligned}
J_{1} & =\int_{S}^{T}-\int_{\Gamma_{1}} u_{i}^{\prime}\left(u \partial_{\nu} u_{i}+(\lambda+\mu) \operatorname{div} u \nu_{i}+\gamma \frac{\partial u_{i}}{\partial x_{i}} \nu_{i}\right) \\
& =\int_{S}^{T}-\int_{\Gamma_{1}} u_{i}^{\prime}\left(-a u_{i}-\lg \left(u_{i}^{\prime}\right)\right) \\
& =\int_{S}^{T} \int_{\Gamma_{1}} u_{i}^{\prime}\left(a u_{i}+\lg \left(u_{i}^{\prime}\right)\right)
\end{aligned}
$$

Substituyendo, obtenemos

$$
\begin{aligned}
0=\int_{S}^{T} & \int_{\Omega} u_{i}^{\prime} u_{i}^{\prime \prime}+\mu \nabla u_{i}^{\prime} \nabla u_{i}+(\lambda+\mu) \operatorname{div} u \frac{\partial u_{i}^{\prime}}{\partial x_{i}}+\gamma \frac{\partial u_{i}}{\partial x_{i}} \frac{\partial u_{i}^{\prime}}{\partial x_{i}} \\
& +\int_{S}^{T} \int_{\Gamma_{1}} u_{i}^{\prime} a u_{i}+l u_{i}^{\prime} g\left(u_{i}^{\prime}\right)
\end{aligned}
$$


Sumando

$$
\begin{aligned}
0= & \int_{S}^{T} \int_{\Omega} u^{\prime} u^{\prime \prime}+\mu \nabla u^{\prime} \nabla u+(\lambda+\mu) \operatorname{div} u \operatorname{div} u^{\prime}+\gamma \sum_{i=1}^{N} \frac{\partial u_{i}}{\partial x_{i}} \frac{\partial u_{i}^{\prime}}{\partial x_{i}} \\
& +\int_{S}^{T} \int_{\Gamma_{1}} u^{\prime} a u+\sum_{i=1}^{N} l u_{i}^{\prime} g\left(u_{i}^{\prime}\right) \\
= & \int_{S}^{T} \int_{\Omega} \frac{\partial}{\partial t} \frac{1}{2}\left\{u^{\prime 2}+\mu|\nabla u|^{2}+(\lambda+\mu)(\operatorname{div} u)^{2}+\gamma \sum_{i=1}^{N}\left(\frac{\partial u_{i}}{\partial x_{i}}\right)^{2}\right\} \\
& +\int_{S}^{T} \int_{\Gamma_{1}} \frac{\partial}{\partial t}\left(\frac{a u^{2}}{2}\right)+\sum_{i=1}^{N} \int_{S}^{T} \int_{\gamma_{1}} l u_{i}^{\prime} g\left(u_{i}^{\prime}\right) .
\end{aligned}
$$

De la definición de $E$ tenemos

$$
0=\int_{S}^{T} \frac{\partial}{\partial t} E(t)+\sum_{i=1}^{N} \int_{S}^{T} \int_{\gamma_{1}} l u_{i}^{\prime} g\left(u_{i}^{\prime}\right)
$$

Luego,

$$
E(S)-E(T)=\sum_{i=1}^{N} \int_{S}^{T} \int_{\gamma_{1}} l \underbrace{u_{i}^{\prime} g\left(u_{i}^{\prime}\right)}_{\geq 0} .
$$

Para simplificar los cálculos, consideremos el vector $M(u) \in \mathbb{R}^{N}$, que tiene por componentes:

$$
\begin{aligned}
M(u)_{i}=M\left(u_{i}\right) & :=2 m_{k} u_{i, k}+(N-1) u_{i} \\
& =2 \sum_{k=1}^{N}\left(m_{k} u_{i, k}\right)+(N-1) u_{i}
\end{aligned}
$$

donde $m=\left(m_{1}, \ldots, m_{N}\right)$.

Para el primer caso suponemos que $g$ satisfaga (23) y (24). Por otro lado, para el segundo caso, cuando $g$ satisface (26), basta tomar $p=1$ en el siguiente resultado.

El siguiente Lema es fundamental en la prueba del resultado de estabilizacion de la energía.

Lema 4.3. Para todo $0 \leq S<T<+\infty$ tenemos:

$$
\begin{aligned}
& 2 \int_{S}^{T} E(t)^{\frac{p+1}{2}} d t=\frac{p-1}{2} \int_{S}^{T} E(t)^{\frac{p-3}{2}} E^{\prime} \int_{\Omega} u^{\prime} M(u) d x d t \\
+ & \int_{S}^{T} E^{\frac{p-1}{2}} \int_{\Gamma}\left(\mu \partial_{\nu} u_{i}+(\lambda+\mu) \operatorname{divu\nu }_{i}+\gamma u_{i, i} \nu_{i}\right) \cdot M\left(u_{i}\right)+a u^{2} d s d t \\
+ & \int_{S}^{T} E^{\frac{p-1}{2}} \int_{\Gamma} m \cdot \nu\left(u^{\prime 2}-\mu|\nabla u|^{2}-(\lambda+\mu)(\operatorname{divu})^{2}-\gamma u_{i, i}^{2}\right) d s d t \\
- & {\left[E^{\frac{p-1}{2}} \int_{\Omega} u^{\prime} \cdot M(u) d x\right]_{S}^{T} }
\end{aligned}
$$


Prueba. Multiplicando la ecuación (8) por $2 m \cdot \nabla u_{i}=2 \sum_{k=1}^{N} m_{k} u_{i, k}$, usando la convención de los indices repetidos, e integrando sobre $\Omega$, para luego multiplicar el resultado por $E^{\frac{p-1}{2}}$ e integrar por partes sobre $[S, T]$, obtenemos

$$
\begin{aligned}
0= & \int_{S}^{T} E^{\frac{p-1}{2}}(t) \int_{\Omega}\left(u_{i}^{\prime \prime}-\mu \Delta u_{i}-(\lambda+\mu) \frac{\partial}{\partial x_{i}}(\operatorname{div} u)-\gamma \frac{\partial^{2} u_{i}}{\partial x_{i}{ }^{2}}\right) 2 m_{k} u_{i, k}+ \\
= & {\left[E^{\frac{p-1}{2}} \int_{\Omega} 2 u_{i}^{\prime} m_{k} u_{i, k}\right]_{S}^{T}-\frac{p-1}{2} \int_{S}^{T} E^{\frac{p-3}{2}} E^{\prime} \int_{\Omega} 2 m_{k} u_{i, k} u_{i}^{\prime} } \\
& \int_{S}^{T} E^{\frac{p-1}{2}} \int_{\Omega}-2 u_{i}^{\prime} m_{k} u_{i, k}^{\prime}+\mu \nabla u_{i} \cdot \nabla\left(2 m_{k} u_{i, k}\right)+ \\
& (\lambda+\mu)(\operatorname{div} u) \frac{\partial}{\partial x_{i}}\left(2 m_{k} u_{i, k}\right)+\gamma u_{i, i} \frac{\partial}{\partial x_{i}}\left(2 m_{k} u_{i, k}\right) \\
& -\int_{S}^{T} E^{\frac{p-1}{2}} \int_{\Gamma}\left(\mu \partial_{\nu} u_{i}+(\lambda+\mu) \operatorname{div} u \nu_{i}+\gamma u_{i, i} \nu_{i}\right) 2 m_{k} u_{i, k}
\end{aligned}
$$

y observando que $\int_{\Omega} m_{k}\left(u_{i}^{\prime 2}\right)_{, k}=-\int_{\Omega} m_{k, k}\left(u_{i}^{\prime}\right)^{2}+\int_{\Gamma} m_{k} u_{i}^{\prime} u_{i}^{\prime} \nu_{k}$ y $m_{k, j}=\delta_{k, j}$, entonces,

$$
\begin{aligned}
& -\left[E^{\frac{p-1}{2}} \int_{\Omega} 2 u_{i}^{\prime} m_{k} u_{i, k}\right]_{S}^{T} \\
& +\int_{S}^{T} E^{\frac{p-1}{2}} \int_{\Gamma}\left(\mu \partial_{\nu} u_{i}+(\lambda+\mu) d i v u \nu_{i}+\gamma u_{i, i} \nu_{i}\right) 2 m_{k} u_{i, k} \\
& =-\frac{p-1}{2} \int_{S}^{T} E^{\frac{p-3}{2}} E^{\prime} \int_{\Omega} 2 m_{k} u_{i, k} u_{i}^{\prime} \\
& +\int_{S}^{T} E^{\frac{p-1}{2}} \int_{\Omega}-m_{k}\left(u_{i}^{\prime 2}\right)_{, k}+\mu 2 u_{i, j} m_{k, j} u_{i, k}+\mu 2 u_{i, j} m_{k} u_{i k, j} \\
& +(\lambda+\mu)(\operatorname{div} u)\left(2 m_{k, i} u_{i, k}+2 m_{k} u_{i k, i}\right)+\gamma u_{i, i}(2 m_{k, i} u_{i, k}+2 m_{k} u_{i} \underbrace{k, i}_{i, k}) \\
& \quad+\int_{S}^{T} E^{\frac{p-1}{2}} \int_{\Omega} N u^{\prime 2}+2 \mu u_{i, j} u_{i, j}+\underbrace{\mu m_{k}\left(u_{i, j}^{2}\right)_{k}}_{S_{1}:=}+(\lambda+\mu)(d i v u) 2 u_{i, i} \\
& +\int_{S}^{T} E^{\frac{p-1}{2}} \int_{\Omega}(\lambda+\mu) \underbrace{m_{k}(\operatorname{div} u)_{, k}^{2}}_{S_{2}:=}+2 \gamma u_{i, i} u_{i, i}+\gamma \underbrace{m_{k}\left(u_{i, i}^{2}\right)_{, k}}_{S_{3}:=} \\
& -\int_{S}^{T} E^{\frac{p-1}{2}} \int_{\Gamma} m_{k} \nu_{k} u_{i}^{\prime} u_{i}^{\prime}
\end{aligned}
$$

$$
\begin{aligned}
S_{1} & =-\int_{\Omega} \sum_{k} \mu \underbrace{m_{k, k}}_{=1}\left(u_{i, j}^{2}\right)+\mu \int_{\Gamma} \sum_{k} m_{k} u_{i, j}^{2} \nu_{k} \\
& =-\mu N \int_{\Omega} u_{i, j}^{2}+\mu \int_{\Gamma} m \cdot \nu u_{i, j}^{2} \\
& =-\mu N \int_{\Omega}|\nabla U|^{2}+\int_{\Gamma} m \cdot \nu|\nabla U|^{2}
\end{aligned}
$$




$$
\begin{aligned}
S_{2} & =-\int_{\Omega} m_{k, k}(\operatorname{div} u)^{2}+\int_{\Gamma} m_{k}(\operatorname{div} u)^{2} \nu_{k} \\
& =-N \int_{\Omega}(\operatorname{div} u)^{2}+\int_{\Gamma} m \cdot \nu(\operatorname{divu})^{2} \\
S_{3} & =-\int_{\Omega} m_{k, k}\left(u_{i, i}^{2}\right)+\int_{\Gamma} m_{k} u_{i, i}^{2} \nu_{k} \\
& =-N \int_{\Omega}\left(u_{i, i}^{2}\right)+\int_{\Gamma} m \cdot \nu u_{i, i}^{2}
\end{aligned}
$$

Substituyendo $S_{1}, S_{2}$ y $S_{3}$ en la identidad previa, tenemos

$$
\begin{aligned}
& -\left[E^{\frac{p-1}{2}} \int_{\Omega} 2 u_{i}^{\prime} m_{k} u_{i, k}\right]_{S}^{T} \\
& +\int_{S}^{T} E^{\frac{p-1}{2}} \int_{\Gamma}\left(\mu \partial_{\nu} u_{i}+(\lambda+\mu) \operatorname{div} u \nu_{i}+\gamma u_{i, i} \nu_{i}\right) 2 m_{k} u_{i, k} \\
& =\int_{S}^{T} E^{\frac{p-1}{2}} \int_{\Omega} N u^{\prime 2}+(2-N) \mu|\nabla u|+(2-N)(\lambda+\mu)(\operatorname{div} u)^{2}+(2-N) \gamma u_{i, i}^{2} \\
& -\int_{S}^{T} E^{\frac{p-1}{2}} \int_{\Gamma}\left(u^{\prime 2}-\mu|\nabla u|-(\lambda+\mu)(\operatorname{div} u)^{2}-\gamma u_{i, i}^{2}\right) m \cdot \nu \\
& -\frac{p-1}{2} \int_{S}^{T} E^{\frac{p-3}{2}} E^{\prime} \int_{\Omega} 2 m_{k} u_{i, k} u_{i}^{\prime}
\end{aligned}
$$

Ahora multiplicamos la ecuación (8) por $u_{i}$ e integrando por partes sobre $\Omega \times[S, T]$, obtenemos:

$$
\begin{gathered}
0=\int_{S}^{T} E^{\frac{p-1}{2}} \int_{\Omega}\left(u_{i}^{\prime \prime}-\mu \Delta u_{i}-(\lambda+\mu) \frac{\partial}{\partial x_{i}}(\operatorname{div} u)-\gamma \frac{\partial^{2} u_{i}}{\partial x_{i}^{2}}\right) u_{i} \\
S_{4}=\int_{S}^{T} E^{\frac{p-1}{2}} \int_{\Omega} u_{i}^{\prime \prime} u_{i} \\
=-\int_{S}^{T} \int_{\Omega}\left(E^{\frac{p-1}{2}} u_{i}\right)^{\prime} u_{i}^{\prime}+\left.E^{\frac{p-1}{2}} \int_{\Omega} u_{i}^{\prime} u\right|_{S} ^{T} \\
=-\left\{\int_{S}^{T} \frac{p-1}{2} E^{\frac{p-3}{2}} E^{\prime} \int_{\Omega} u_{i} u_{i}^{\prime}+\int_{S}^{T} E^{\frac{p-1}{2}} \int_{\Omega} u_{i}^{\prime} u_{i}^{\prime}\right\}+\left.E^{\frac{p-1}{2}} \int_{\Omega} u^{\prime} u\right|_{S} ^{T} \\
=-\frac{p-1}{2} \int_{S}^{T} E^{\frac{p-3}{2}} E^{\prime} \int_{\Omega} u_{i} u_{i}^{\prime}-\int_{S}^{T} E^{\frac{p-1}{2}} \int_{\Omega}\left(u_{i}^{\prime}\right)^{2}+\left.E^{\frac{p-1}{2}} \int_{\Omega} u^{\prime} u\right|_{S} ^{T} \\
S_{5}=-\mu \int_{S}^{T} E^{\frac{p-1}{2}} \int_{\Omega} \Delta u_{i} u_{i} \\
=\mu \int_{S}^{T} E^{\frac{p-1}{2}}\left[\int_{\Omega} \nabla u_{i} \nabla u_{i}-\int_{\Gamma} u_{i} \frac{\partial u_{i}}{\partial \nu}\right] \\
=\mu \int_{S}^{T} E^{\frac{p-1}{2}} \int_{\Omega}|\nabla u|^{2}-\mu \int_{S}^{T} E^{\frac{p-1}{2}} \int_{\Gamma} u_{i} \frac{\partial u_{i}}{\partial \nu}
\end{gathered}
$$




$$
\begin{aligned}
S_{6} & =-\int_{S}^{T} E^{\frac{p-1}{2}} \int_{\Omega} \frac{\partial}{\partial x_{i}}(\operatorname{div} u) u_{i} \\
& =\int_{S}^{T} E^{\frac{p-1}{2}} \int_{\Omega}(\operatorname{div} u) \frac{\partial}{\partial x_{i}} u_{i}-\int_{S}^{T} E^{\frac{p-1}{2}} \int_{\Gamma} u_{i} \operatorname{div} u \nu_{i} \\
& =\int_{S}^{T} E^{\frac{p-1}{2}} \int_{\Omega}(\operatorname{div} u)^{2}-\int_{S}^{T} E^{\frac{p-1}{2}} \int_{\Gamma} u_{i} \operatorname{div} u \nu_{i} \\
& S_{7}=\int_{S}^{T} E^{\frac{p-1}{2}} \int_{\Omega} \frac{\partial^{2} u_{i}}{\partial x_{i}^{2}} u_{i} \\
= & \int_{S}^{T} E^{\frac{p-1}{2}}\left\{-\int_{\Omega} \frac{\partial u_{i}}{\partial x_{i}} \frac{\partial u_{i}}{\partial x_{i}}+\int_{\Gamma} \frac{\partial u_{i}}{\partial x_{i}} u_{i} \nu_{i}\right\}
\end{aligned}
$$

Usando $S_{4}, S_{5}, S_{6}$ y $S_{7}$ en (40) obtenemos,

$$
\begin{aligned}
& -\left[E^{\frac{p-1}{2}} \int_{\Omega} u^{\prime} u\right]_{S}^{T}+\int_{S}^{T} E^{\frac{p-1}{2}} \int_{\Gamma} u_{i}\left\{\mu \partial_{\nu} u_{i}+(\lambda+\mu) \operatorname{div} u \nu_{i}+\gamma u_{i, i} \nu_{i}\right\} \\
= & \int_{S}^{T} E^{\frac{p-1}{2}} \int_{\Omega}\left\{-u^{\prime 2}+\mu|\nabla u|^{2}+(\lambda+\mu)(\operatorname{div} u)^{2}+\gamma u_{i, i}^{2}\right\} \\
& -\frac{p-1}{2} \int_{S}^{T} E^{\frac{p-3}{2}} E^{\prime} \int_{\Omega} u_{i}^{\prime} u_{i} .
\end{aligned}
$$

Al resultado de multiplicar (41) por $N$ - 1, le adicionamos (39), obtendremos la identidad (38).

Ahora vamos ha mayorar la identidad (38), usando las condiciones de frontera sobre $\Gamma_{0}$ y sobre $\Gamma_{1}$. Esto conseguimos en los siguientes Lemas.

\section{Lema 4.4.}

$$
\begin{aligned}
& J:=\int_{\Gamma_{0}} \underbrace{\left(\mu \partial_{\nu} u_{i}+(\lambda+\mu) d i v u \nu_{i}+\gamma u_{i, i} \nu_{i}\right) \cdot M\left(u_{i}\right)}_{J_{1}:=}+\underbrace{a u^{2}}_{=0} d s \\
& +\int_{\Gamma_{0}} m \cdot \nu\left(u^{\prime 2}-\mu|\nabla u|^{2}-(\lambda+\mu)(\operatorname{divu})^{2}-\gamma u_{i, i}^{2}\right) d s \leq 0
\end{aligned}
$$

\section{Prueba.}

Sobre $\Gamma_{0}$ tenemos que $u_{i}=0$, entonces

a) $\nabla u_{i}=\left(\partial_{\nu} u_{i}\right) \nu \rightarrow|\nabla u|^{2}=\sum_{i=1}^{N}\left(\partial_{\nu} u_{i}\right)^{2}$. $\left(\right.$ i.e. $\left.u_{i, k}=\left(\partial_{\nu} u_{i}\right) \nu_{k}\right) \rightarrow u_{i, i}=\operatorname{div} u$.

b)

$$
\begin{aligned}
M\left(u_{i}\right) & :=2 m_{k} u_{i, k}+(N-1) \underbrace{u_{i}}_{=0} \\
& =2 m_{k} u_{i, k} \\
& =2 m_{k}\left(\partial_{\nu} u_{i}\right) \nu_{k} \\
& =2\left(\partial_{\nu} u_{i}\right) m \cdot \nu
\end{aligned}
$$


Observamos que

$$
\begin{aligned}
J_{1} & =2\left(\partial_{\nu} u_{i}\right) m \cdot \nu\left(\mu \partial_{\nu} u_{i}+(\lambda+\mu) \operatorname{divu\nu }_{i}+\gamma u_{i, i} \nu_{i}\right) \\
& =[2 \mu\left(\partial_{\nu} u_{i}\right)^{2}+2(\lambda+\mu) \operatorname{divu} \underbrace{\nu_{i}\left(\partial_{\nu} u_{i}\right)}_{=\text {div } u}+2 \gamma u_{i, i} \underbrace{\nu_{i}\left(\partial_{\nu} u_{i}\right)}_{=u_{i, i}}] m \cdot \nu
\end{aligned}
$$

luego,

$$
\begin{aligned}
J & =\int_{\Gamma_{0}} \underbrace{m \cdot \nu}_{\leq 0}\left(u^{\prime 2}+\mu|\nabla u|^{2}+(\lambda+\mu)(\operatorname{div} u)^{2}+\gamma u_{i, i}^{2}\right) \\
& \leq 0
\end{aligned}
$$

Supongamos que $g$ satisface (26) y $a$ y $l$ estan definidas por (28), entonces se obtienen las siguientes estimativas.

Lema 4.5.

$$
\begin{aligned}
& \int_{\Gamma_{1}}\left(\mu \partial_{\nu} u_{i}+(\lambda+\mu) d i v u \nu_{i}+\gamma u_{i, i} \nu_{i}\right) \cdot M\left(u_{i}\right)+\tilde{a} u^{2} d s \\
& +\int_{\Gamma_{1}} m \cdot \nu\left(u^{\prime 2}-\mu|\nabla u|^{2}-(\lambda+\mu)(\operatorname{divu})^{2}-\gamma u_{i, i}^{2}\right) d s \\
& \leq \frac{2 R}{\sqrt{\xi}} \frac{c^{\prime}}{c} \int_{\Gamma_{1}} \tilde{l} u_{i}^{\prime} g\left(u_{i}^{\prime}\right) d s
\end{aligned}
$$

Lema 4.6. Se satisface la siguiente desigualdad:

$$
\left|\int_{\Omega} u^{\prime} \cdot M(u) d x\right| \leq \frac{2 R}{\sqrt{\xi}} E(t)
$$

Finalmente, usando las estimativas (42), (43) y (44) y eligiendo $p=1$ en la desigualdad (38) obtenemos:

$$
\begin{aligned}
2 \int_{S}^{T} E(r) d r & \leq \frac{2 R}{\sqrt{\xi}}\left(1+\frac{c^{\prime}}{c}\right) E(S)+\frac{2 R}{\sqrt{\xi}}\left(1-\frac{c^{\prime}}{c}\right) E(T) \\
\int_{S}^{\infty} E(r) d r & \leq \frac{R}{\sqrt{\xi}}\left(1+\frac{c^{\prime}}{c}\right) E(S), \quad \forall S \geq 0
\end{aligned}
$$

Aplicando el Lema (2.1) obtenemos que

$$
E(S) \leq E(0) e^{1-\frac{c \sqrt{\xi}}{\left(c+c^{\prime}\right) R} S}, \quad \forall S \geq 0 .
$$

Por otro lado, supongamos que $g$ satisface (26) y $a$ y $l$ estan definidas en (22), entonces

Lema 4.7. Existe una constante $C>0$ tal que

$$
\begin{aligned}
& \int_{\Gamma_{1}}\left(u^{\prime 2}-\mu|\nabla u|^{2}-(\lambda+\mu)(\operatorname{div} u)^{2}-\gamma u_{i, i}^{2}\right) m \cdot \nu \\
& +\int_{\Gamma_{1}}\left(\mu \partial_{\nu} u_{i}+(\lambda+\mu) \operatorname{div} u \nu_{i}+\gamma u_{i, i} \nu_{i}\right) \cdot M\left(u_{i}\right)+a u^{2} \\
& \quad \leq C \int_{\Gamma_{1}} l u_{i}^{\prime} g\left(u_{i}^{\prime}\right)+a u_{i} u_{i}, \\
& \left|\int_{\Omega} u^{\prime} \cdot M(u)\right| \leq C E(t) .
\end{aligned}
$$


Utilizando (42), (46) y (47) obtenemos

$$
2 \int_{S}^{T} E(\tau) d \tau \leq 2 C E(S)+C \int_{S}^{T} \int_{\Gamma_{1}} a u^{2}
$$

El siguiente resultado es una adaptación del método de F. Conrad y B. Rao.

Lema 4.8. Existe una constante $C>0$ tal que, para todo $\epsilon>0$ vale

$$
\int_{S}^{T} \int_{\Gamma_{1}} a u^{2} d s d t \leq \frac{C}{\epsilon} E(s)+\epsilon \int_{S}^{T} E(\tau) d \tau
$$

En la desigualdad (49) consideramos $\epsilon$ suficientemente pequeño de modo que la desigualdad (48), nos conduce a

$$
\int_{S}^{\infty} E(\tau) d \tau \leq C_{5} E(S)
$$

Luego por Lema 2.1, obtenemos el resultado.

Para el caso $g$ satisfaciendo (23), (24) y a y $l$ definidos por (22), oḅtenemos la siguiente estimativa

$$
\exists C>0 \text { tal que } \int_{S}^{+\infty} E^{\frac{p+1}{2}} \leq C E(S)
$$

La desigualdad integral dada en [7] página 124, nos dice que si $E$ es una función no creciente que satisface (50), entonces

$$
E(t) \leq C(E(0)) t^{\frac{-2}{p-1}}, \forall t \geq 0
$$

donde $C$ es una función continua de $E(0)$. 


\section{REFERENCIAS BIBLIOGRÁFICAS}

[1] Barbu, V. Analysis and control of nonlinear infinite dimensional systems, Academic Press , New York, 1993.

[2] Ciarlet, P. G. Matehematical elasticity, Vol. 1: Three-dimensional elasticity, North-Holland, amsterdam, 1988.

[3] Conrad, F.and Rao, B. Decay of solution of wave equations in a star-shaped domain with nonlinear boundary feedback. Asymptot Anal, 7 1993, 159-177.

[4] Dafermos, C.M. Asymptotic behaviour of solutions of evolution equations in Nonlinear Evolution equations, M.G. Crandall Ed. Academic Press, New YorK, 1978,103-123.

[5] Dautry, R. and Lions, J.L. analyse mathematique et calcul scientifique pour les sciences et les techniques. Tome I, Chapitre 7, Masson Paris,1988.

[6] Feynman, R. P. , Leyton and Sands The Feynman's Lectures on Physics, Vol 2, chapter 39, Addison Wesley, 1964.

[7] Komornik, V. Exact controllability and stabilization, RAM : Research in applied mathematics, Masson Paris 1994.

[8] Komornik, V. Boundary stabilization of isotropic elasticity systems, Lectures Notes in Pure and, Dekker New York Applied Mathematics.174, 1995, 135-146.

[9] Lagnese, J.E. Boundary stabilization of linear elastodynamic systems, SIAM J. Control Optim. $21,1983,968-984$.

[10] Lagnese, J.E. Uniform asymptotic energy estimates for solutions of the equations of dynamic plane elasticity with nonlinear dissipation at the boundary, nonlinear Anal. , 16; 1991, 35 -54.

[11] Lions, J. L. Quelque methodes de resolutions de problemes aux limites non lineares, Dunod Paris. 1969.

[12] Liu, W. Partial exact controllability and exponential satability in higher -dimencional linear thermoelasticity, Esaim: Control, Opt. and calc. of Var,Vol 3 ,1998, 23-48.

[13] Pazy, A. Semigroups of linear operator and applications to partial differential equations. Applied Mathematical Sciences. 44 Springer Verlag, Berlin,1983.

[14] Russel, D. L. Controllability and stabilizability theory for linear partial differential equations.Recent progress and open questions. SIAM Rev. 20, 1978 , 639-739.

[15] Santiago, Yolanda About decay of solution of the wave equation with dissipation. PROYECCIONES Journal of Mathematics, Vol. 26, Nro. 1, pp 37-71, May 2007. 
[16] Santiago, Yolanda Decaimiento exponencial de la solución débil de una ecuación de la Onda no lineal. PESQUIMAT Revista de la Fac. CC. MM. de la UNMSM. Vol VIII, Nro. 2, Diciembre 2005. pag $29-43$.

[17] Santiago, Yolanda Estabilidad exponencial del Semigrupo $C_{0}$ asociado a un sistema Termoelástico. PESQUIMAT Revista de la Fac. CC. MM. de la UNMSM. Vol VII , Nro. 1, Junio 2004, pág 30-42.

[18] Santiago, Yolanda Global existence and exponential decay to the wave equation with localized frictional damping. PESQUIMAT Revista de la Fac. CC. MM. de la UNMSM. Vol V. Nro. 2, Diciembre 2002, pág 1-19.

[19] R. Showalter. Hilbert Space Methods for Partial Differential Equations . London: Pitman, (1977) 